DOI: https://doi.org/10.24297/jac.v16i0.8476

\title{
Clitocybins and 2-Substituted-Isoindolin-1-Ones: Synthesis and in Vitro Antimycobacterial Activities
}

\author{
Amani Mejai ${ }^{a,}{ }^{\S}$ Nambinina V. Rakotoarivelo ${ }^{b, c}, \S$, Ennaji Najahi ${ }^{a}, b, c^{*}$, Pierre Perio ${ }^{b, c}$ Etienne Hollande ${ }^{d}$, Maria \\ Rosalia Pasca e, Rym Abidi a, Françoise Nepveu ${ }^{b, c}$ \\ aUniversité Carthage, Faculté des sciences de Bizerte, LACReSNE, Interactions Moléculaires Spécifiques, \\ Zarzouna-Bizerte,Tunis TN 7021,Tunisie.
}

bUniversité de Toulouse III, UPS, PHARMA-DEV, UMR 152, 118 Route de Narbonne, F-31062 Toulouse cedex 9 , France.

IRD, UMR 152, F-31062 Toulouse cedex 9, France.

dINSERM UMR-1037, Université de Toulouse, Cancer Research Center of Toulouse (CRCT), Equipe Labellisée ligue contre le cancer and Laboratoire d'Excellence Toulouse Cancer (TOUCAN), Toulouse, France.

eUniversity of Pavia, Dipartimento di Biologia e Biotecnologie "Lazarro Spallanzani", via Ferrata 1, 27100 Pavia, Italy.

ennaji.najahi@univ-tlse3.fr

\begin{abstract}
Despite strong indications of antimycobacterial activities for clitocybins reported since 1945, no reports linking chemical structure and activity have been reported in the literature since then. In this study, we synthesized some clitocybin derivatives (also called 2-substituted-isoindolinones), and tested their activities and carried out some chemical derivations. Isoindolinones were prepared from methyl 2-formyl-3,5-dimethoxybenzoate and various primary aromatic amines. Compounds were evaluated for in vitro activity against Mycobacterium tuberculosis $\mathrm{H}_{37} \mathrm{Rv}$, as well as for cytotoxicity $\left(\mathrm{CC}_{50}\right)$ on the Vero cell line. 4,6-dihydroxy-2-(4hydroxyphenyl)isoindolin-1-one, 7, and 4-hydroxy-2-(4-hydroxyphenyl)-6-methoxy-isoindol-1-one, 5, exhibited the highest antimycobacterial activities (minimum inhibitory concentration $=19.45 \mu \mathrm{M}$ and 18.45 $\mu \mathrm{M}$, respectively) and were non-toxic ( $\mathrm{CC}_{50}=30 \mu \mathrm{M}$ and $29 \mu \mathrm{M}$, respectively).
\end{abstract}

Keywords: Isoindolinone, Tuberculosis, Clitocybins, Cytotoxicity, Antibiotics.

\section{Introduction}

Tuberculosis (TB), is still a leading cause of death in developing countries and a resurgent disease in developed countries [1]. Because of the development and increasing spread of Mycobacterium tuberculosis $(M t b)$ drug resistant strains, new drugs with novel mode of action are needed.

Our starting point to find new lead compounds was the antitubercular activity of clitocybins reported over several decades. In 1945, A. Ch Hollande highlighted for the first time in a higher fungus, the presence of a powerful antibiotic in Clitocybe gigantea, a basidiomycetes mushroom of the family Agaricaceae [2]. He showed the antibiotic activity against a large number of Gram-positive and Gram-negative bacteria, of a compound to which he gave the name "clitocybin".

He also reported the bacteriostatic and bacteriolytic action of the acid clitocybin against tuberculosis bacilli [3]. A few years later, A. Ch Hollande extracted clitocybin in the form of crystals and he considered that it contained two substances, clitocybin A, which was active against Staphylococcus spp. and inactive against the tuberculosis bacilli, and clitocybin $B$, that was very active against the tuberculosis bacilli but only slightly 
against Staphylococcus spp. [4]. A.F. Rebert showed that the lamellae and spores of the C. gigantea contained the most active elements [5]. Subsequently, in collaboration with A. Ch Hollande, he established that clitocybin was a glycoside extracted from mycelia [6]. Assuming that

clitocybin would be present in the mycelial cells, J.C. Ringenbach isolated from the mycelia of C. gigantea a substance he called "Proclitocybine" [7]. Analysis of this substance, despite its extreme lability, showed that it corresponded to a glycoside form containing sulfur and that it liberated as pentose and an aglycone by hydrolysis [7]. Its bactericide properties were comparable to the clitocybin isolated by A. Ch Hollande, but its action was more gradual, longer-lasting and less toxic to the animal. Finally clitocybin was shown to be an antimitotic agent [7]. This early work certainly highlighted bactericide and antimitotic endocellular substances in the spores and mycelia of C. gigantea. Since then, many antibiotic substances have been isolated from several species and families of fungi.

Anchel et al. isolated "lilludine", an antibiotic substance from the Clitocybe illudens that was considered to be different from the clitocybin of A. Ch Hollande [8]. Müller-Stoll et al. demonstrated the antibiotic activity of clitocybin isolated from Leucopaxillus gigantéa and from Clitocybe nebularis [9]. More recently some authors have extracted molecules that have various activities from other Clitocybe species. Pohleven et al. have characterized and purified an antiproliferative molecule against human T leukemia cells from Clitocybe nebularis [10]. Moon et al. isolated a substance that inhibits cell death by apoptosis and cell senescence from Clitocybe aurantiaca [11].

Kim et al. reported a new molecule with antioxidant activity they call "clitocybin A" isolated from the same upper mushroom [12] and then they isolated a new cytotoxic isoindolinone alkaloid from Hericium erinaceum [13]. This molecule was considered as a new isoindoline that inhibited the cell proliferation of vascular smooth muscle by regulating the PI3K/Akt cascade [14] and smooth muscle cell proliferation through suppressing PDGF-RB phosphorylation [15]. Finally, Kim et al. (2009) isolated an enzyme inhibitor of human neutrophil elastase they called "Clitocybin D". [16]

Despite strong indications of antimycobacterial activities for clitocybins reported since 1945, no reports linking their chemical structure and activity have been reported in the literature since then. In this study, we synthesized some clitocybin derivatives, tested their activities and also carried out some chemical derivations (Figure 1). We started from the chemical structure described by Kim et al [12]. The minimum inhibitory concentration (MIC) was determined against Mycobacterium tuberculosis $\mathrm{H}_{37} \mathrm{Rv}$ and cytotoxicity was evaluated using Vero cells in culture.

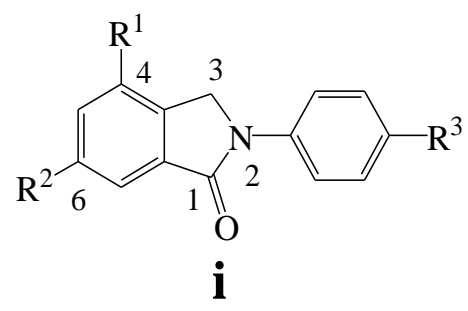

Figure 1: Structure of isoindolinone derivatives $\mathbf{i}$ (i: compounds from 1 to 11).

\section{Results and discussion}

\subsection{Synthetic chemistry}

Isoindolinone derivatives were obtained using methods developed by Yoo et al. [17] (Scheme 1). The reaction between methyl 2-formyl-3,5-dimethoxybenzoate a with the appropriate amines in methanol was carried at room temperature over $1 \mathrm{~h}$. The resulting solution was mixed with sodium borohydride at $0{ }^{\circ} \mathrm{C}$ and reacted overnight at room temperature to produce isoindolinone compounds $\mathbf{i}$ (Table 1). 
<smiles>[Y]N1C=C2C(=O)C(OCC)=CC(OC)=C2C1</smiles>

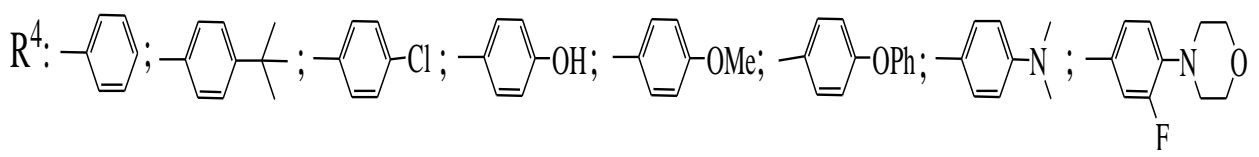

Scheme 1: Synthesis of 2-substituted-iso indolin-1-ones. Reagents and conditions: (i) $\mathrm{NaBH}_{4}, \mathrm{MeOH} 0^{\circ} \mathrm{C}$-r.t., $20 \mathrm{~h}$

The demethylated compounds were prepared according to the procedure previously reported [17]. The two methoxy groups of isoindolinone $\mathbf{4}$ were converted to hydroxyl groups by reflux with acetic acid and bromic acid and compounds 5, $\mathbf{6}$ and $\mathbf{7}$ were obtained (Scheme 2). We isolated the new isoindolinone, 5, which is an isomer of compound $\mathbf{6}$ and has not been previously reported [17]. Its presence was demonstrated by the LCMS spectra, where peaks with two different retention times $(9$ and $9.5 \mathrm{~min})$ and with the same $[\mathrm{M}+\mathrm{H}]^{+}$peaks were observed (see supporting information).

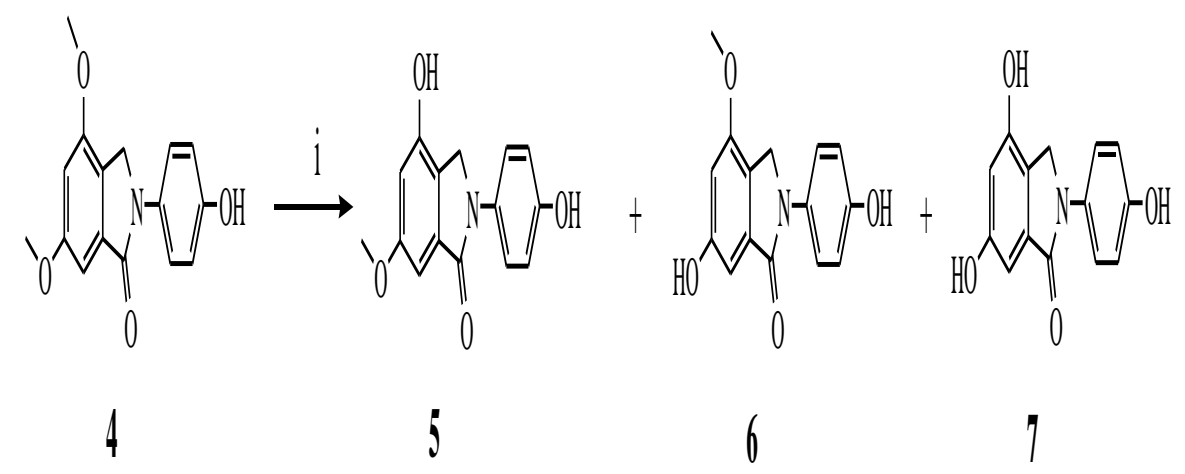

Scheme 2: Demethylation of the isoindolinone 4. (i): $48 \% \mathrm{HBr} /$ acetic acid (1/2), reflux, $18 \mathrm{~h}$.

All the compounds were characterized by chromatography (TLC and HPLC), infrared (IR), NMR $\left({ }^{1} \mathrm{H}\right.$ and $\left.{ }^{13} \mathrm{C}\right)$, and mass spectrometry as well as HRMS. The structures of the isoindolinones were determined primarily from spectroscopic data. The IR spectra showed bands in the range of $1700-1680 \mathrm{~cm}^{-1}$ which confirmed the presence of a $\mathrm{C}=\mathrm{O}$ function. The ${ }^{13} \mathrm{C}-\mathrm{NMR}$ spectra confirmed the formation of the isoindolinone ring with signals of the $C=O$ function at $\delta=166-168 \mathrm{ppm}$ and $\mathrm{CH}_{2}$ at the $\delta=47.90-48.26 \mathrm{ppm}$.

The mass spectra showed the respective $[\mathrm{M}+\mathrm{H}]^{+}$peaks. Log $P_{\text {calc }}$ values (Table 1) calculated with the VCCLAB software [18] ranged between 1.20 and 4.24 with compound $\mathbf{2}$ being the most lipophilic in the series, and $\mathbf{7}$, the least.

\subsection{Cytotoxicity and antitubercular activity}

All compounds were assessed for their in vitro cytotoxicity/antitubercular activity against the Vero cell line and M. tuberculosis, respectively. The cytotoxicity/antitubercular potency of the compounds was compared with the standard drugs doxorubicin and isoniazid. The $50 \%$ cytotoxic concentration $\left(\mathrm{CC}_{50}\right)$ and the minimum inhibitory concentration (MIC) values were calculated as summarized in Table 1. 
Table 1. Structure and biological activity of isoindolinones 1-11.

\begin{tabular}{|c|c|c|c|c|}
\hline Compounds & Structure & $\log \mathbf{P}_{\text {calc }^{b}}$ & MIC $(\mu \mathrm{M})$ & $\mathrm{CC}_{50}(\mu \mathrm{M})$ Vero cellsc \\
\hline 1 & & 2.34 & $>74$ & $>37$ \\
\hline 2 & & 4.24 & $>62$ & $>30$ \\
\hline 3 & & 3.05 & $>66$ & $>33$ \\
\hline $4^{a}$ & & 2.47 & 70.17 & 17 \\
\hline 5 & & 1.65 & 18.45 & 29 \\
\hline $6^{a}$ & & 1.67 & 27.67 & 18 \\
\hline $7^{a}$ & & 1.20 & 19.45 & 30 \\
\hline 8 & & 2.51 & $>67$ & 33 \\
\hline 9 & & 4.00 & $>55$ & $>28$ \\
\hline 10 & & 2.54 & $>64$ & 26 \\
\hline 11 & & 2.64 & $>54$ & nd \\
\hline Isoniazid & & 0.70 & 0.36 & nd \\
\hline Doxorubicin & & nd & nd & $5.12 \pm 0.17$ \\
\hline
\end{tabular}

${ }^{a}$ Ref [17] ; ${ }^{b} \log P_{\text {calc; }}$ calculated with VCCLAB (http://www.virtuallaboratory.org/lab/alogps/start.html); ${ }^{c}$ The drug concentration needed to cause a $50 \%$ decrease in the cell viability. $\mathbf{4}=$ clitocybin $\mathrm{C} ; \mathbf{6}=$ clitocybin $\mathrm{B} ; \mathbf{7}=$ clitocybin A. 
The most active compounds were 5, $\mathbf{6}$ and $\mathbf{7}(\mathrm{MIC} / \mu \mathrm{M}: \mathbf{5}=18.45, \mathbf{6}=27.67$ and $\mathbf{7}=19.45)$. Substituents with different lipophilicity were introduced at $R^{3}$ of the molecule. The activities decreased when the lipophilicity increased: the most active compounds were then the most polar. The presence of at least one hydroxyl group at $R^{1}$ or $R^{2}$ is necessary to obtain a good activity with a hydroxyl group at $R^{3}$. A decrease in the activity is observed when two or three hydroxyl groups are replaced by methoxy groups as well as when various substituents are introduced at $R^{3}$ of the molecules. The cytotoxicity of isoindolinones was relatively low and within the same range as that of doxorubicin $\left(C_{50}=5.12 \pm 0.17 \mu \mathrm{M}\right)$.

\section{Conclusion}

In conclusion, eleven isoindolinones have been prepared and evaluated against $M$. tuberculosis strain $\mathrm{H}_{37} \mathrm{Rv}$. Only the isoindolinones with hydroxyl groups had moderate antimycobacterial activity, with the best one being the isoindolinone $5(\mathrm{MIC}=18.45 \mu \mathrm{M})$. Initial attempts of pharmacomodulation did not improve the activities since two hydroxyl groups or three or at least one on each phenyl group were necessary to maintain activity. For the first time we have established the relationship between the chemical structures of clitocybin derivatives and their antitubercular activity described by A. Ch Hollande sixty years ago.

\section{Experimental}

\subsection{Chemistry}

\subsubsection{General}

Melting points were determined with an Electrothermal 9300 capillary melting point apparatus and are uncorrected. IR spectra were recorded on a Perkin-Elmer PARAGON $1000 \mathrm{FT}$-IR spectrometer. ${ }^{1} \mathrm{H}$ and ${ }^{13} \mathrm{C}$ NMR spectra were recorded on an $\mathrm{AC}$ Bruker spectrometer at $300 \mathrm{MHz}\left({ }^{1} \mathrm{H}\right)$ and $75 \mathrm{MHz}\left({ }^{13} \mathrm{C}\right)$ using $\left(\mathrm{CD}_{3}\right)_{2} \mathrm{SO}$ or $\mathrm{CDCl}_{3}$ as solvents. High resolution mass spectra (HRMS) were recorded on a Bruker Maxis spectrometer (Service Commun Toulouse, France). Preparative HPLC was done using an X Bridge C18 column (Service commun d'HPLC, ICT, Toulouse, France). Silica Gel 60 (Merck 70-230) was used for column chromatography. The progress of the reactions was monitored by thin layer chromatography (TLC) on Kieselgel 60 F254 (Merck) plates. Compound purity was determined by an LC-PDA-MS method and was found to be in the range 96$99 \%$.

\subsubsection{General experimental procedure for the preparation of 2-substituted-isoindolin-1-ones}

To a solution of methyl 2-formyl-3,5-dimethoxybenzoate $(2.5 \mathrm{mmol})$ in methanol $(20 \mathrm{~mL})$ was added the appropriate amine $(2.5 \mathrm{mmol})$. The mixture was stirred at room temperature until the appearance of a suspension. $\mathrm{NaBH}_{4}(5 \mathrm{mmol})$ was then added at $0{ }^{\circ} \mathrm{C}$ and the mixture allowed to react at room temperature for $20 \mathrm{~h}$ with stirring. The precipitate thus formed was filtered through a glass filter to give a solid compound.

4.1.2.1. 4,6-dimethoxy-2-phenylisoindolin-1-one (1). White solid, yield: $62 \%, \mathrm{mp}: 181-183{ }^{\circ} \mathrm{C} . \mathrm{IR}(\mathrm{KBr}) \mathrm{cm}^{-1}$ : 3067, 2910, 2836, 1692 (C=O), 1617, 1596, 1501, 1451, 1383, 1328, 1211, 1141, 1115, 1056, 948, 816, 758, 693, 632, 520, 505. ${ }^{1} \mathrm{H}$ NMR $\left(300 \mathrm{MHz}\right.$, DMSO- $\left.d_{6}\right) \delta: 3.86\left(\mathrm{~s}, 3 \mathrm{H}, \mathrm{CH}_{3}\right), 3,90\left(\mathrm{~s}, 3 \mathrm{H}, \mathrm{CH}_{3}\right), 4.86\left(\mathrm{~s}, 2 \mathrm{H}, \mathrm{CH}_{2}\right), 6.83(\mathrm{~d}, J=$ $3 \mathrm{~Hz}, 1 \mathrm{H}), 6.87(\mathrm{~d}, J=3 \mathrm{~Hz}, 1 \mathrm{H}), 7.15-7.20(\mathrm{~m}, 1 \mathrm{H}), 7.40-7.46(\mathrm{~m}, 2 \mathrm{H}), 7.90-7.93(\mathrm{~m}, 2 \mathrm{H}) .{ }^{13} \mathrm{C} \mathrm{NMR}(75 \mathrm{MHz}$, DMSO-d $\left.d_{6}\right)$ d: $48.4\left(\mathrm{CH}_{2}\right), 56.19\left(\mathrm{CH}_{3}\right), 56.23\left(\mathrm{CH}_{3}\right), 98.4(\mathrm{CH}), 103.5(\mathrm{CH}), 119.8(2 \mathrm{CH}), 121.8(\mathrm{C}), 124.5(\mathrm{CH})$, $129.4(2 \mathrm{CH}), 135.0$ (C), 139.9 (C), 155.5 (C), 162.0 (C), 167.0 (C=O). MS-(+)ESI m/z: 270 ([M+H] $\left.{ }^{+}, 100 \%\right)$. HRMSESI (m/z): calcd for $\mathrm{C}_{16} \mathrm{H}_{16} \mathrm{NO}_{3}[\mathrm{M}+\mathrm{H}]^{+} 270.1130$ found: 270.1133 .

4.1.2.2. 2-(4-tert-butylphenyl)-4,6-dimethoxyisoindolin-1-one (2). White solid, yield: $61 \%, \mathrm{mp}: 194-196{ }^{\circ} \mathrm{C}$. IR $(\mathrm{KBr}) \mathrm{cm}^{-1}:$ 2965, 2945, 2904, 2866, 1695 (C=O), 1627, 1615, 1522, 1503, 1452, 1384, 1336, 1263, 1238, 1208, $1147,1108,1051,949,842,819,764,558,518 .{ }^{1} \mathrm{H}$ NMR $\left(300 \mathrm{MHz}, \mathrm{DMSO}-d_{6}\right) \delta: 1.30\left(\mathrm{~s}, 9 \mathrm{H}, 3 \mathrm{CH}_{3}\right), 3.85(\mathrm{~s}, 3 \mathrm{H}$, $\left.\mathrm{CH}_{3}\right), 3.90\left(\mathrm{~s}, 3 \mathrm{H}, \mathrm{CH}_{3}\right), 4.82\left(\mathrm{~s}, 2 \mathrm{H}, \mathrm{CH}_{2}\right), 6.82(\mathrm{~d}, J=3 \mathrm{~Hz}, 1 \mathrm{H}), 6.86(\mathrm{~d}, J=3 \mathrm{~Hz}, 1 \mathrm{H}), 7.43(\mathrm{~d}, J=9 \mathrm{~Hz}, 2 \mathrm{H}), 7.81$ $(\mathrm{d}, J=9 \mathrm{~Hz}, 2 \mathrm{H}) .{ }^{13} \mathrm{C}$ NMR $\left(75 \mathrm{MHz}, \mathrm{DMSO}-d_{6}\right) \delta: 31.6\left(3 \mathrm{CH}_{3}\right), 34.5(\mathrm{C}), 48.4\left(\mathrm{CH}_{2}\right), 56.16\left(\mathrm{CH}_{3}\right), 56.22\left(\mathrm{CH}_{3}\right), 98.4$ 
$(\mathrm{CH}), 103.3(\mathrm{CH}), 119.6(2 \mathrm{CH}), 121.8(\mathrm{C}), 126.0(2 \mathrm{CH}), 135.1(\mathrm{C}), 137.4(\mathrm{C}), 147.0(\mathrm{C}), 155.4(\mathrm{C}), 162.0(\mathrm{C}), 166.8$ (C). MS-(+)ESI m/z: $326\left([M+H]^{+}, 100 \%\right)$. HRMS-ESI (m/z): calcd for $\mathrm{C}_{20} \mathrm{H}_{24} \mathrm{NO}_{3}[\mathrm{M}+\mathrm{H}]^{+} 326.1756$ found: 326.1760 .

4.1.2.3. 2-(4-chlorophenyl)-4,6-dimethoxyisoindolin-1-one (3). White solid, yield: $65 \%$, mp: $186-188{ }^{\circ} \mathrm{C}$. IR (KBr) $\mathrm{cm}^{-1}:$ 2975, 2911, 2840, 1698 (C=O), 1632 1610, 1505, 1494, 1456, 1381, 1364, 1320, 1211, 1144, 1114, 1049, 935, 821, 798, 762, 626, 505. ${ }^{1} \mathrm{H}$ NMR (300 MHz, DMSO-d $)$ \&: $3.85\left(\mathrm{~s}, 3 \mathrm{H}, \mathrm{CH}_{3}\right), 3.90\left(\mathrm{~s}, 3 \mathrm{H}_{1} \mathrm{CH}_{3}\right), 4.84(\mathrm{~s}, 2 \mathrm{H}$, $\left.\mathrm{CH}_{2}\right), 6.83(\mathrm{~d}, J=3 \mathrm{~Hz}, 1 \mathrm{H}), 6.87(\mathrm{~d}, J=3 \mathrm{~Hz}, 1 \mathrm{H}), 7.46-7.49(\mathrm{~m}, 2 \mathrm{H}), 7.94-7.96(\mathrm{~m}, 2 \mathrm{H}) .{ }^{13} \mathrm{C} \mathrm{NMR}(75 \mathrm{MHz}$, DMSO-d $\left.d_{6}\right)$ d: $47.9\left(\mathrm{CH}_{2}\right), 55.71\left(\mathrm{CH}_{3}\right), 55.74\left(\mathrm{CH}_{3}\right), 97.9(\mathrm{CH}), 103.2(\mathrm{CH}), 120.7(2 \mathrm{CH}), 121.3(\mathrm{C}), 127.8(\mathrm{C}), 128.7$

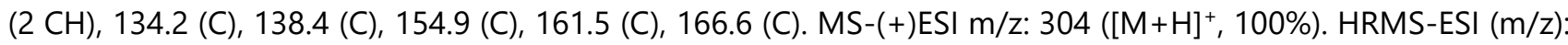
calcd for $\mathrm{C}_{16} \mathrm{H}_{15} \mathrm{CINO}_{3}[\mathrm{M}+\mathrm{H}]^{+} 304.0740$ found: 304.0735 .

4.1.2.4. 4,6-dimethoxy-2-(4-methoxyphenyl)isoindolin-1-one (8). White solid, yield: $51 \%, \mathrm{mp}: 141-143{ }^{\circ} \mathrm{C}$. IR (KBr) $\mathrm{cm}^{-1}:$ 3076, 3001, 2918, 2836, 1679 (C=O), 1615, 1513, 1505, 1451, 1385, 1327, 1211, 1144, 1110, 1055, 949, 814, 767, 586, 511; ${ }^{1} \mathrm{H}$ NMR (300 MHz, DMSO- $\left.d_{6}\right) \delta: 3.77\left(\mathrm{~s}, 3 \mathrm{H}, \mathrm{CH}_{3}\right), 3.85\left(\mathrm{~s}, 3 \mathrm{H}_{1} \mathrm{CH}_{3}\right), 3.89\left(\mathrm{~s}, 3 \mathrm{H}, \mathrm{CH}_{3}\right)$, $4.80\left(\mathrm{~s}, 2 \mathrm{H}, \mathrm{CH}_{2}\right), 6.80(\mathrm{~d}, J=3 \mathrm{~Hz}, 1 \mathrm{H}), 6.85(\mathrm{~d}, J=3 \mathrm{~Hz}, 1 \mathrm{H}), 6.98-7.01(\mathrm{~m}, 2 \mathrm{H}), 7.77-7.80(\mathrm{~m}, 2 \mathrm{H}) .{ }^{13} \mathrm{C} \mathrm{NMR}(75$ $\mathrm{MHz}$, DMSO-d $\left.d_{6}\right)$ \&: $48.7\left(\mathrm{CH}_{2}\right), 55.7\left(\mathrm{CH}_{3}\right), 56.15\left(\mathrm{CH}_{3}\right), 56.21\left(\mathrm{CH}_{3}\right), 98.4(\mathrm{CH}), 103.2(\mathrm{CH}), 114.5(2 \mathrm{CH}), 121.67$ (C), 121.70 (2 CH), 133.1 (C), 135.1 (C), 155.4 (C), 156.4 (C), 162.0 (C), 166.5 (C). MS-(+)ESI m/z: 300 ([M+H] ${ }^{+}$, 100\%). HRMS-ESI (m/z): calcd for $\mathrm{C}_{17} \mathrm{H}_{18} \mathrm{NO}_{4}[\mathrm{M}+\mathrm{H}]^{+} 300.1236$ found: 300.1231 .

4.1.2.5. 4,6-dimethoxy-2-(4-phenoxyphenyl)isoindolin-1-one (9). White solid, yield: $51 \%, \mathrm{mp}: 149-151{ }^{\circ} \mathrm{C}$. IR $(\mathrm{KBr}) \mathrm{cm}^{-1}:$ 3005, 2963, 2932, 2840, 1685 (C=O), 1627, 1506, 1488, 1451, 1385, 1363, 1336, 1237, 1207, 1146, $1118,1051,1043,934,835,781,696,560,518 ;{ }^{1} \mathrm{H}$ NMR $\left(300 \mathrm{MHz}, \mathrm{DMSO}-d_{6}\right) \delta: 3.86\left(\mathrm{~s}, 3 \mathrm{H}_{1} \mathrm{CH}_{3}\right), 3.90(\mathrm{~s}, 3 \mathrm{H}$, $\left.\mathrm{CH}_{3}\right), 4.84\left(\mathrm{~s}, 2 \mathrm{H}, \mathrm{CH}_{2}\right), 6.82(\mathrm{~d}, J=3 \mathrm{~Hz}, 1 \mathrm{H}), 6.87(\mathrm{~d}, J=3 \mathrm{~Hz}, 1 \mathrm{H}), 7.00-7.04(\mathrm{~m}, 2 \mathrm{H}), 7.08-7.11(\mathrm{~m}, 2 \mathrm{H}), 7.13-$ $7.16(\mathrm{~m}, 1 \mathrm{H}), 7.37-7.42(\mathrm{~m}, 2 \mathrm{H}), 7.91-7.94(\mathrm{~m}, 2 \mathrm{H}) .{ }^{13} \mathrm{C} \mathrm{NMR}\left(75 \mathrm{MHz}, \mathrm{DMSO}-d_{6}\right)$ 8: $48.6\left(\mathrm{CH}_{2}\right), 56.18\left(\mathrm{CH}_{3}\right), 56.23$ $\left(\mathrm{CH}_{3}\right), 98.4(\mathrm{CH}), 103.4(\mathrm{CH}), 118.6(2 \mathrm{CH}), 119.8(2 \mathrm{CH}), 121.6(2 \mathrm{CH}), 121.8(\mathrm{C}), 123.7(\mathrm{CH}), 130.5(2 \mathrm{CH}), 134.9$

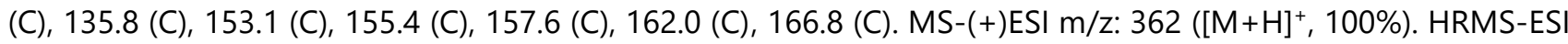
(m/z): calcd for $\mathrm{C}_{22} \mathrm{H}_{20} \mathrm{NO}_{4}[\mathrm{M}+\mathrm{H}]^{+} 362.1392$ found: 362.1385 .

4.1.2.6. 2-(4-(dimethylamino)phenyl)-4,6-dimethoxyisoindolin-1-one (10). White solid, yield 64\%, $\mathrm{mp}$ : 152-153 ${ }^{\circ} \mathrm{C}$. IR (KBr) cm $\mathrm{cm}^{-1}:$ 1692, 1683 (C=O), 1614, 1522, 1326, 1270, 1207, 1146, 1110, 1050, 944, 808. ${ }^{1} \mathrm{H}$ NMR (300 $\left.\mathrm{MHz}, \mathrm{DMSO}-\mathrm{d}_{6}\right) \delta: 2.89\left(\mathrm{~s}, 6 \mathrm{H}, \mathrm{CH}_{3}\right), 3.84\left(\mathrm{~s}, 3 \mathrm{H}, \mathrm{CH}_{3}\right), 3.88\left(\mathrm{~s}, 3 \mathrm{H}, \mathrm{CH}_{3}\right), 4.74\left(\mathrm{~s}, 2 \mathrm{H}, \mathrm{CH}_{2}\right), 6.76-6.79(\mathrm{~m}, 3 \mathrm{H}), 6.83$ $(\mathrm{d}, J=3 \mathrm{~Hz}, 1 \mathrm{H}), 7.64-7.67(\mathrm{~m}, 2 \mathrm{H}) .{ }^{13} \mathrm{C}$ NMR $\left(75 \mathrm{MHz}, \mathrm{DMSO}-d_{6}\right) \delta: 40.8\left(2 \mathrm{CH}_{3}\right), 48.8\left(\mathrm{CH}_{2}\right), 56.1\left(\mathrm{CH}_{3}\right), 56.2$ $\left(\mathrm{CH}_{3}\right), 98.3(\mathrm{CH}), 102.9(\mathrm{CH}), 113.1(2 \mathrm{CH}), 121.5(\mathrm{C}), 121.6(2 \mathrm{CH}), 129.6(\mathrm{C}), 135.4(\mathrm{C}), 148.1(\mathrm{C}), 155.4(\mathrm{C}), 161.9$ (C), 166.3 (C). MS-(+)ESI m/z: $313\left([\mathrm{M}+\mathrm{H}]^{+}, 100 \%\right)$. HRMS-ESI (m/z): calcd for $\mathrm{C}_{18} \mathrm{H}_{21} \mathrm{~N}_{2} \mathrm{O}_{3}[\mathrm{M}+\mathrm{H}]^{+} 313.1552$ found: 313.1551 .

4.1.2.7. 2-(3-fluoro-4-morpholinophenyl)-4,6-dimethoxyisoindolin-1-one (11). White solid, yield 79\%, $\mathrm{mp}$ : 204$205^{\circ} \mathrm{C}$. IR (KBr) cm $\mathrm{cm}^{-1}:$ 2949, 2840, 1688 (C=O), 1627, 1512, 1363, 1328, 1270, 1204, 1146, 1129, 1118, 1054, 1042, 935, 824. ${ }^{1} \mathrm{H}$ NMR $\left(300 \mathrm{MHz}, \mathrm{CDCl}_{3}\right) \delta: 3.10\left(\mathrm{t}, J=3 \mathrm{~Hz}, 4 \mathrm{H}, \mathrm{CH}_{2}\right), 3.88\left(\mathrm{t}, J=3 \mathrm{~Hz}, 4 \mathrm{H}, \mathrm{CH}_{2}\right), 3.89(\mathrm{~s}, 3 \mathrm{H}$, $\left.\mathrm{CH}_{3}\right), 3.90\left(\mathrm{~s}, 3 \mathrm{H}, \mathrm{CH}_{3}\right), 4.67\left(\mathrm{~s}, 2 \mathrm{H}, \mathrm{CH}_{2}\right), 6.64(\mathrm{~d}, J=3 \mathrm{~Hz}, 1 \mathrm{H}), 6.95-7.01(\mathrm{~m}, 2 \mathrm{H}), 7.48(\mathrm{dd}, J=3,9 \mathrm{~Hz}, 1 \mathrm{H}), 7.79$ $(\mathrm{dd}, J=3,9 \mathrm{~Hz}, 1 \mathrm{H}) .{ }^{13} \mathrm{C} \mathrm{NMR}\left(75 \mathrm{MHz}, \mathrm{CDCl}_{3}\right) \delta: 48.4\left(\mathrm{CH}_{2}\right), 51.03\left(\mathrm{CH}_{2}\right), 51.07\left(\mathrm{CH}_{2}\right), 55.6\left(\mathrm{CH}_{3}\right), 55.9\left(\mathrm{CH}_{3}\right)$, $67.0\left(2 \mathrm{CH}_{2}\right), 97.9(\mathrm{CH}), 103.0(\mathrm{CH}), 108.4(\mathrm{CH}), 114.7(\mathrm{CH}), 118.8(\mathrm{CH}), 121.1(\mathrm{C}), 135.1(\mathrm{C}), 136.4(\mathrm{C}), 153.9(\mathrm{C})$, 155.1 (C), 157.1 (C), 162.0 (C), 167.3 (C); MS-(+)ESI m/z: $373\left([M+H]^{+}, 100 \%\right)$. HRMS-ESI m/z: calcd for $\mathrm{C}_{20} \mathrm{H}_{22} \mathrm{FN}_{2} \mathrm{O}_{4}[\mathrm{M}+\mathrm{H}]^{+} 373.1564$ found: 373.1555 .

4.1.2.8. Preparation of 4-hydroxy-2-(4-hydroxyphenyl)-6-methoxy-isoindol-1-one (5). A 48\% aqueous bromic acid solution $(10 \mathrm{ml})$ and acetic acid $(20 \mathrm{ml})$ were added to the isoindolinone $4(2.0 \mathrm{~g}, 7.0 \mathrm{mmol})$, and were heated for $18 \mathrm{~h}$ with reflux. The resulting reaction was mixed ice water, followed by ethyl acetate. The organic layer thus formed was washed with water, dried over anhydrous sodium sulfate, concentrated in a vacuum and purified through silica gel column chromatography (dichloromethane/methanol: 10/1) to produce the compounds $\mathbf{7}$ and the mixture of $\mathbf{5}$ and $\mathbf{6}$. The compounds $\mathbf{5}$ and $\mathbf{6}$ were separated by preparative HPLC. White solid, yield $2 \%$, mp: 147-149 ${ }^{\circ} \mathrm{C}$. IR ( $\left.\mathrm{KBr}\right) \mathrm{cm}^{-1}$ : 3398, 2928, 2840, 1685 (C=O), 1616, 1512, 1431, 1374, 
1319, 1268, 1228, 1146, 1069, 1042. ${ }^{1} \mathrm{H}$ NMR $\left(300 \mathrm{MHz}_{\mathrm{CDCl}}\right)$ ) $\mathrm{C}: 3.90(\mathrm{~s}, 3 \mathrm{H}), 4.64\left(\mathrm{~s}, 2 \mathrm{H}, \mathrm{CH}_{2}\right), 6.65-6.74(\mathrm{~m}$, $4 \mathrm{H}), 7.61(\mathrm{~d}, J=9 \mathrm{~Hz}, 2 \mathrm{H}), 9.38(\mathrm{~s}, 1 \mathrm{H}) .{ }^{13} \mathrm{C} \mathrm{NMR}\left(75 \mathrm{MHz}, \mathrm{CDCl}_{3}\right)$ 8: $48.6\left(\mathrm{CH}_{2}\right), 55.7\left(\mathrm{CH}_{3}\right), 100.6(\mathrm{CH}), 103.1(2$ $\mathrm{CH}), 115.0(\mathrm{CH}), 120.4(\mathrm{C}), 121.7(2 \mathrm{CH}), 133.1(\mathrm{C}), 135.0(\mathrm{C}), 154.5(\mathrm{C}), 158.8(\mathrm{C}), 161.5(\mathrm{C}), 166.3(\mathrm{C})$; MS-(+)ESI m/z: $272\left([\mathrm{M}+\mathrm{H}]^{+}, 100 \%\right)$. HRMS-ESI m/z: calcd for $\mathrm{C}_{15} \mathrm{H}_{14} \mathrm{NO}_{4}[\mathrm{M}+\mathrm{H}]^{+} 272.0923$ found: 272.0952 .

\subsection{Biology}

\subsubsection{Inhibition of mycobacterial growth in vitro}

The minimum inhibitory concentration (MIC) was determined to evaluate the susceptibility of the $M$. tuberculosis strain $\mathrm{H}_{37} \mathrm{Rv}$ to all the synthesized compounds. M. tuberculosis $\mathrm{H}_{37} \mathrm{Rv}$ strain was grown either in Middlebrook $7 \mathrm{H} 9$ broth (Difco) supplemented with $0.05 \%$ Tween 80 , or on Middlebrook $7 \mathrm{H} 11$ agar (Difco) supplemented with $0.5 \%$ glycerol, both supplemented with $10 \%(\mathrm{v} / \mathrm{v})$ OADC. All the test compounds were dissolved in dimethyl sulfoxide (DMSO). A single colony of $M$. tuberculosis strain was used to inoculate complete Middlebrook $7 \mathrm{H} 9$ both. The cultures were incubated at $37^{\circ} \mathrm{C}$ until the exponential growth phase (about $10^{8} \mathrm{CFU} / \mathrm{ml}$ ) was reached, corresponding to an $\mathrm{OD}_{600}$ ranging from 0.8 to 1.0 . Cultures were diluted to a final concentration of about $10^{7} \mathrm{CFU} / \mathrm{ml} ; 1 \cdot$ I of the diluted cultures was then streaked onto plates containing two-fold serial dilutions of the appropriate compound. MIC values were scored as the lowest drug concentrations inhibiting bacterial growth. The MIC of isoniazid was determined as control. All assays were repeated three times.

\subsubsection{In vitro cytotoxicity assay: Vero cells}

Cytotoxicity was estimated on Vero cells. The cells were cultured in RPMI 1640 medium (BioWhittaker, Cambrex, Belgium) containing L-glutamine (BioWhittaker), $25 \mathrm{mM}$ HEPES (BioWhittaker), and 10\% fetal calf serum (Cambrex). After trypsinization, the cells were distributed in 96-well plates at $2 \times 10^{4}$ cells/well in $100 \mu \mathrm{l}$ of culture medium added to $100 \mu \mathrm{l}$ of the same medium containing the test compounds at various concentrations (the final concentrations in the wells were 1,10 and $100 \mu \mathrm{g} / \mathrm{ml}$ ). Cell growth was estimated by $\left[{ }^{3} \mathrm{H}\right]$-hypoxanthine incorporation following a $48 \mathrm{~h}$ incubation and was compared with a control sample that did not have the added test compounds (the mean of the corresponding wells was referred to as $100 \%$ ) [19].

\section{References}

1. WHO: Global tuberculosis report 2013: http://www.who.int/tb/publications/global report/en/index.html

2. Ch. Hollande, Mass lysis of Koch's bacilli in the guinea-pig after treatment with clitocybin. Inhibitory property of this product in respect of the typhus bacillus, the coli bacillus, Brucella abortus, C., C. R. Acad. Sc. Paris 221 (1945) 361-363.

3. A.Ch. Hollande, La bacteriostase et la bacteriolyse du bacille tuberculeux par la Clitocybine, C. R. Acad. Sc. Paris 224 (1947) 1534-1535.

4. Ch. Hollande, A propos de la clitocybine cristallisée, C. R. Acad. Sc. Paris 228 (1949) 1758-1760.

5. A.F. Rebert, Recherches sur la clitocybine contenue dans les carpophores de clitocybe candida, Doctoral Thesis (Pharmacie), Montpellier, 1949.

6. A.F. Rebert, J.C. Ringenbach, A. Ch. Hollande. Isolement d'un hetroside du mycelium de Clitocybe gigantea. Trav. Soc. Pharm. Montpellier 16 (1956) 161-163.

7. J.C. Ringenbach, Contribution à l'étude du Clitocybe gigantea (Fries ex Sowerby) Gillet et de ces principes antibiotiques, Doctoral Thesis (Pharmacie). Strasbourg 1962, 1-104. 
8. M. Anchel, A. Hervey, W.J. Robbins, Antibiotic substances from Basidiomycetes VII. Clitocybe illudens, Proc. Natl. Acad. Sci. USA. 36 (1950) 300-305.

9. W.R. Müller-Stoll, The antibiotic activity of clitocybine and nebularine from leucopaxillus giganteus and clitocybe nebularis, Zeitschrift Fuer Mykologie 56 (1990) 167-186.

10. J. Pohleven, N. Obermajer, J. Sabotic, S. Anzlovar, K. Sepcić, J. Kos, B. Kralj, B. Strukelj, J. Brzin, Purification, characterization and cloning of a ricin B-like lectin from mushroom Clitocybe nebularis with antiproliferative activity against human leukemic T cells, Biochim. Biophys. Acta. 1790 (2009) 173-181.

11. E.Y. Moon, J.M. Oh, Y.H. Kim, I.J. Ryoo, I.D. Yoo, Clitocybins, novel isoindolinone free radical scavengers, from mushroom Clitocybe aurantiaca inhibit apoptotic cell death and cellular senescence, Biol. Pharm. Bull. 32 (2009) 1689-1694.

12. Y.H. Kim, S.M. Cho, J.W. Hyun, I.J. Ryoo, S.J. Choo, S. Lee, S.J. Seok, J.S. Hwang, E.D. Sohn, B.S. Yun, K.H. Bae, I.D. Yoo. A new antioxidant, clitocybin A, from the culture broth of Clitocybe aurantiaca, J. Antibiot. (Tokyo) 61 (2008) 573-576.

13. K.H. Kim, H.J. Noh, S.U. Choi, K.R. Lee, Isohericenone, a new cytotoxic isoindolinone alkaloid from Hericium erinaceum, J. Antibio. 65 (2012) 575-577.

14. K.D. Yoo, E.S. Park, Y. Lim, S.I. Kang, S.H. Yoo, H.H. Won, Y.H. Kim, I.D. Yoo, H.S. Yoo, J.T. Hong, Y.P. Yun, Clitocybin $A$, a novel isoindolinone, from the mushroom Clitocybe aurantiaca, inhibits cell proliferation through G1 phase arrest by regulating the PI3K/Akt cascade in vascular smooth muscle cells, J. Pharmacol. Sci. 118 (2012), 171-177.

15. K.D. Yoo, E.S. Park, Y. Lim, S.I. Kang, S.H. Yoo, H.H. Won, H.P. Lee, Y.H. Kim, I.D. Yoo, H.S. Yoo, clitocybin B inhibits rat aortic smooth muscle cell proliferation through suppressing PDGF-RB phosphorylation, Vascul. Pharmacol. 56 (2012) 91-97.

16. Y.H. Kim, I.J. Ryoo, S.J. Choo, G.H. Xu, S. Lee, S.J. Seok, K. Bae, I.D. Yoo, Clitocybin D, a novel human neutrophil elastase inhibitor from the culture broth of Clitocybe aurantiaca, J. Microbiol. Biotechnol. 19 (2009) 1139-1141.

17. I.D. Yoo, I.J. Ryoo, Y.H. Kim, S.J. Choo, S. Lee, Novel clitocybin derivatives, preparation method thereof and composition containing the extract of clitocybe aurantiaca Kctc $11143 \mathrm{bp}$ or the novel clitocybin derivatives for prevention of aging as an active ingredient. Korea Res. Inst. Patent WO 2009102083.

18. ALOGPS 21; http://www.virtuallaboratory.org/lab/alogps/; access date 10-11 July 2009.

19. J. Bouquet, M. Rivaud, S. Chevalley, E. Deharo, V. Jullian, A. Valentin, Biological activities of nitidine, a potential anti-malarial lead compound, Malaria J. 11 (2012) 67.

\section{Figure and scheme captions}

Figure 1. Structure of isoindolinone derivatives $\mathbf{i}$ (i: compounds from $\mathbf{1}$ to $\mathbf{1 1}$ ).

Scheme 1. Synthesis of 2-substituted-isoindolin-1-ones. Reagents and conditions: (i) $\mathrm{NaBH}_{4}, \mathrm{MeOH}, 0^{\circ} \mathrm{C}$-r.t., $20 \mathrm{~h}$.

Scheme 2. Demethylation of the isoindolinone 4. (i): $48 \% \mathrm{HBr} /$ acetic acid (1/2), reflux, $18 \mathrm{~h}$. 